\title{
UK Renal Registry 17th Annual Report: Appendix K Renal Centre Names and Abbreviations used in the Figures and Data Tables
}

\section{Adult Centres}

\begin{tabular}{|c|c|c|}
\hline City & Hospital & Abbreviati \\
\hline \multicolumn{3}{|l|}{ England } \\
\hline Basildon & Basildon Hospital & Basldn \\
\hline Birmingham & Heartlands Hospital & B Heart \\
\hline Birmingham & Queen Elizabeth Hospital & B QEH \\
\hline Bradford & St Luke’s Hospital & Bradfd \\
\hline Brighton & Royal Sussex County Hospital & Brightn \\
\hline Bristol & Southmead Hospital & Bristol \\
\hline Cambridge & Addenbrooke’s Hospital & Camb \\
\hline Carlisle & Cumberland Infirmary & Carlis \\
\hline Carshalton & St Helier Hospital & Carsh \\
\hline Chelmsford & Broomfield Hospital & Chelms \\
\hline Colchester & Colchester General Hospital & Colchr \\
\hline Coventry & University Hospital Coventry & Covnt \\
\hline Derby & Royal Derby Hospital & Derby \\
\hline Doncaster & Doncaster Royal Infirmary & Donc \\
\hline Dorset & Dorset County Hospital & Dorset \\
\hline Dudley & Russells Hall Hospital & Dudley \\
\hline Exeter & Royal Devon and Exeter Hospital & Exeter \\
\hline Gloucester & Gloucestershire Royal Hospital & Glouc \\
\hline Hull & Hull Royal Infirmary & Hull \\
\hline Ipswich & Ipswich Hospital & Ipswi \\
\hline Kent & Kent and Canterbury Hospital & Kent \\
\hline Leeds & St James's University Hospital and Leeds General Infirmary & Leeds \\
\hline Leicester & Leicester General Hospital & Leic \\
\hline Liverpool & Aintree University Hospital & Liv Ain \\
\hline Liverpool & Royal Liverpool University Hospital & Liv Roy \\
\hline London & St. Bartholomew's Hospital and The Royal London Hospital & L Barts \\
\hline London & St George’s Hospital and Queen Mary's Hospital & L St. G \\
\hline London & Guy’s Hospital and St Thomas' Hospital & L Guys \\
\hline London & Hammersmith, Charing Cross,St Mary's & L West \\
\hline London & King’s College Hospital & L Kings \\
\hline London & Royal Free, Middlesex and UCL Hospitals & L Rfree \\
\hline Manchester & Manchester Royal Infirmary & M RI \\
\hline Middlesbrough & The James Cook University Hospital & Middlbr \\
\hline Newcastle & Freeman Hospital and Royal Victoria Infirmary & Newc \\
\hline Norwich & Norfolk and Norwich University Hospital & Norwch \\
\hline
\end{tabular}

\section{KARGER 125\%}

Fax +4161306 1234

E-Mail karger@karger.com

www.karger.com
(C) 2015 S. Karger AG, Basel

$1660-8151 / 15 / 1295-0321 \$ 39.50 / 0$

Accessible online at: www.karger.com/nec
UK Renal Registry, Southmead Hospital, Southmead Road, Bristol, BS10 5NB, UK

Email: renalregistry@renalregistry.nhs.uk 


\begin{tabular}{|c|c|c|}
\hline City & Hospital & Abbreviation \\
\hline Nottingham & Nottingham City Hospital & Nottm \\
\hline Oxford & John Radcliffe Hospital and Churchill Hospital & Oxford \\
\hline Portsmouth & Queen Alexandra Hospital & Ports \\
\hline Preston & Royal Preston Hospital & Prestn \\
\hline Reading & Royal Berkshire Hospital & Redng \\
\hline Shrewsbury & Royal Shrewsbury Hospital & Shrew \\
\hline Southend & Southend Hospital & Sthend \\
\hline Stevenage & Lister Hospital & Stevng \\
\hline Stoke & Royal Stoke University Hospital & Stoke \\
\hline Sunderland & Sunderland Royal Hospital & Sund \\
\hline \multicolumn{3}{|l|}{ Wales } \\
\hline Bangor & Ysbyty Gwynedd & Bangor \\
\hline Cardiff & University Hospital of Wales & Cardff \\
\hline Clwyd & Glan Clwyd Hospital & Clwyd \\
\hline Swansea & Morriston Hospital & Swanse \\
\hline Wrexham & Wrexham Maelor Hospital & Wrexm \\
\hline \multicolumn{3}{|l|}{ Scotland } \\
\hline Aberdeen & Aberdeen Royal Infirmary & Abrdn \\
\hline Airdrie & Monklands Hospital & Airdrie \\
\hline Dumfries & Dumfries \& Galloway Royal Infirmary & D \& Gall \\
\hline Dundee & Ninewells Hospital & Dundee \\
\hline Edinburgh & Royal Infirmary of Edinburgh & Edinb \\
\hline Newry & Daisy Hill Hospital & Newry \\
\hline Ulster & Ulster Hospital & Ulster \\
\hline
\end{tabular}

\section{Paediatric Centres}

\begin{tabular}{llll}
\hline City & Hospital & Abbreviation & Country \\
\hline Belfast & Royal Belfast Hospital for Sick Children & Blfst_P & N Ireland \\
Birmingham & Birmingham Children's Hospital & Bham_P & England \\
Bristol & Bristol Royal Hospital for Children & Brstl_P & England \\
Cardiff & KRUF Children's Kidney Centre & Cardf_P & Wales \\
Glasgow & Royal Hospital for Sick Children & Glasg_P & Scotland \\
Leeds & Leeds Children's Hospital & Leeds_P & England \\
Liverpool & Alder Hey Children's Hospital & Livpl_P & England \\
London & Guy's Hospital - Paediatric & L Eve_P & England \\
London & Great Ormond Street Hospital for Children & LGOSH_P & England \\
Manchester & Royal Manchester Children's Hospital & Manch_P & England \\
Newcastle & Great North Children's Hospital & Newc_P & England \\
Nottingham & Nottingham Children's Hospital & Nottm_P & England \\
Southampton & Southampton General Hospital - Paediatric & Soton_P & England \\
\hline
\end{tabular}

